\title{
Advanced control scheme of a unifiedpower flow controller using sliding mode control
}

\author{
Abdellatif Hinda ${ }^{1}$, Mounir Khiat ${ }^{2}$, Zinelaabidine Boudjema ${ }^{3}$ \\ ${ }^{1,2}$ SCAMRE Laboratory, Department of Electrical Engineering, Algeria \\ ${ }^{3}$ Electrical Engineering Department, University of Chlef, Algeria
}

\begin{tabular}{l}
\hline Article Info \\
\hline Article history: \\
Received Apr 10, 2019 \\
Revised Dec 28, 2019 \\
Accepted Jan 23, 2020 \\
\hline
\end{tabular}

Keywords:

FACTS

Sliding mode control power UPFC

\begin{abstract}
This paper presents an advanced control scheme based on sliding mode control of a unified power flow controller (UPFC). This controller can generate a number of benefits in terms of static and dynamic operation of the power system such as the control law is synthesized with two kinds of controllers: sliding mode controller (SMC), and proportional integral (PI). Their respective performances are compared in terms of reference monitoring, sensitivity to disturbances and robustness. We have to study the problem of controlling power in electric system by UPFC. The simulation results show the effectiveness of the proposed strategy especially in chattering-free behavior, response to sudden load variations and robustness. All the simulations for the above work have been carried out using MATLAB/Simulink. Various simulations have given very satisfactory results and we have successfully improved the active and reactive power flows on a line of transmission, as well as to control voltage at the bus where it is connected, the studies and illustrate the effectiveness and capability of UPFC in improving power.
\end{abstract}

This is an open access article under the CC BY-SA license.

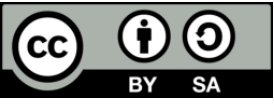

\section{Corresponding Author:}

Abdellatif Hinda,

Department of Electrical Engineering,

Laboratory (SCAMRE) Simulation, Commanded, Analysis and Maintenance Electrical Network.

ENP of Oran, BP 1523 El Mnaouer, Oran, Algeria.

Email: abdellatif.hinda2@gmail.com

\section{INTRODUCTION}

In recent years, the electrical power distribution system are suffering as of significant power flow quality (PQ) problems, which are characterized using low power factor, poor voltage quality, voltage stability, supply interruptions, and load unbalancing. These power profile issues have attracted attention to the researchers in the academic and industrial sectors. This difficulty and augment in power supply needs providing the system with recent control systems which contribute to the improvement of the electrical grids performances [1]. The use of flexible AC transmission system (FACTS) controllers in power system has been of worldwide interest for enhancing power system controllability and stability and increasing the power transfer capability due to their flexibility and speed. In addition, converter-based FACTS controllers are capable of independently controlling the active and reactive power flow in the power system [2].

UPFC is the member of FACTS device. It is the mainly adaptable and powerful FACTS device [3]. The fundamental theory of UPFC is that, the magnitude of voltage affects flow of reactive power and the phase angle affects flow of real power [4, 5]. The UPFC consists of two other FACTS devices: the Static Synchronous Compensator (STATCOM) and the Static Synchronous Series Compensator (SSSC), the SSSC injects a an almost sinusoidal voltage, of variable magnitude in series using the system voltage which 
provides the majority cost effective solution to mitigate voltage sags through improving power quality level that is required with a customer. While, the STATCOM is joined or connected with a common DC link capacitor. It can, at the same time, to UPFC bus voltage/shunt reactive power control, in addition perform the function of transmission line real/reactive power flow control [6].

Though UPFC implies many advantages, but its controller design still being a matter of challenge since it is a multi-variable controller. In literature, many works have been presented by different control schemes of UPFC for various power system applications. Recently, the sliding mode control method has been extensively employed for control of nonlinear systems. Several papers have been published based on SMC of UPFC [7-9]. The classical SMC is super in terms of parameters variation, response time, external disturbances. In addition, it ensures convergence in finite time and it is very simple to be implemented in any low cost materieal [10].

This article discusses the competence of UPFC on controlling independently active and reactive powers into the power transmission line, the improvement of the transient and dynamic stability of the power system using the UPFC. Using two kind of controllers PI and SMC, active and reactive powers are controlled. Their performances are analysed and compared in terms of reference tracking, sensitivity to robustness plus perturbations.

\section{STRUCTURE OF THE UPFC}

For many years, UPFC is considered as the most versatile of the FACTS device one that can be used to enhance steady state stability, dynamic stability and transient stability, which combines the good features of STATCOM and SSSC. Figure 1 shows the basic structure of UPFC which is consists of two voltage sourced converters (VSC) and employed to give galvanic isolation and regulate the voltage levels in the supply system. It is consisted of two PWM (Pulse Width Modulation) inverters, which are operated from a DC link provided by a DC storage capacitor. The first inverter is coupled in parallel while the second one is placed in series with the transmission line [11]. The detailed structure and the functionality of the UPFC can be found in [12].

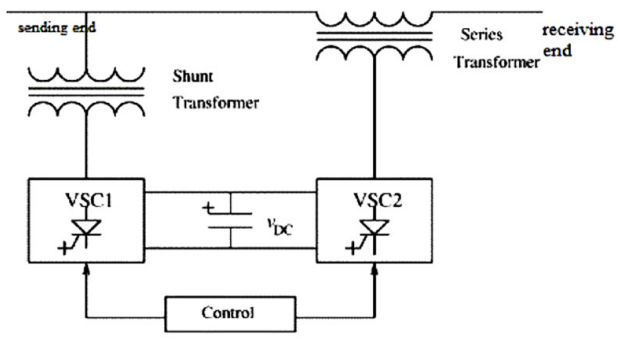

Figure 1. Basic structure of the UPFC

\section{THE UPFC MODEL}

Figure 2 represents the simplified model circuit of the UPFC. The UPFC mathematical model can be divided into three sets of equations, namely: the equations of the parallel branch, the equations of the series branch and those of the continuous one.

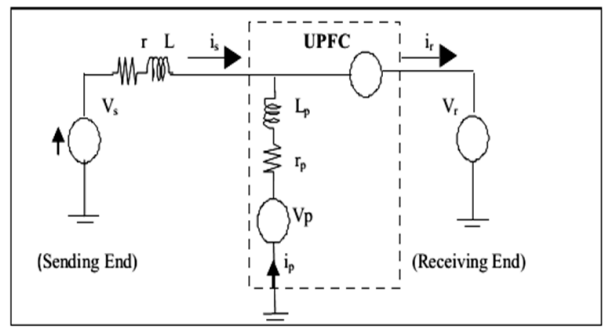

Figure 2. Equivalent circuit of the UPFC

Int J Pow Elec \& Dri Syst, Vol. 11, No. 2, June 2020 : 625 - 633 

equations

By applying Kirchhoff's law on the circuit of Figure 2, we can write the following system of

$$
\frac{d}{d t}\left[\begin{array}{c}
I_{s a} \\
I_{s b} \\
I_{s c}
\end{array}\right]=\left[\begin{array}{ccc}
\frac{-r}{L} & 0 & 0 \\
0 & \frac{-r}{L} & 0 \\
0 & 0 & \frac{-r}{L}
\end{array}\right]\left[\begin{array}{c}
I_{s a} \\
I_{s b} \\
I_{s c}
\end{array}\right]+\left[\begin{array}{ccc}
\frac{1}{L} & 0 & 0 \\
0 & \frac{1}{L} & 0 \\
0 & 0 & \frac{1}{L}
\end{array}\right]\left[\begin{array}{c}
V_{s a}-V_{c a}-V_{r a} \\
V_{s b}-V_{c b}-V_{r b} \\
V_{s c}-V_{c c}-V_{r c}
\end{array}\right]
$$

By applying Park's transformation to the system (1), we can write:

$$
\frac{d}{d t}\left[\begin{array}{l}
I_{s d} \\
I_{s q}
\end{array}\right]=\left[\begin{array}{cc}
-r / L & +\omega \\
-\omega & -r / L
\end{array}\right]\left[\begin{array}{l}
I_{s d} \\
I_{s q}
\end{array}\right]+\left[\begin{array}{cc}
1 / L & 0 \\
0 & 1 / L
\end{array}\right]\left[\begin{array}{l}
V_{s d}-V_{c d}-V_{r d} \\
V_{s q}-V_{c q}-V_{r q}
\end{array}\right]
$$

\subsection{Modeling of the UPFC shunt branch}

The complete mathematical model of the UPFC shunt can be established similarly using the following matrix

$$
\frac{d}{d t}\left[\begin{array}{c}
I_{p a} \\
I_{p b} \\
I_{p c}
\end{array}\right]=\left[\begin{array}{ccc}
\frac{-r_{p}}{L_{p}} & 0 & 0 \\
0 & \frac{-r_{p}}{L_{p}} & 0 \\
0 & 0 & \frac{-r_{p}}{L_{p}}
\end{array}\right]\left[\begin{array}{c}
I_{p a} \\
I_{p b} \\
I_{p c}
\end{array}\right]+\left[\begin{array}{ccc}
\frac{1}{L_{p}} & 0 & 0 \\
0 & \frac{1}{L_{p}} & 0 \\
0 & 0 & \frac{1}{L_{p}}
\end{array}\right]\left[\begin{array}{c}
V_{p a}-V_{c a}-V_{r a} \\
V_{p b}-V_{c b}-V_{r b} \\
V_{p c}-V_{c c}-V_{r c}
\end{array}\right]
$$

Since the system is assumed to be a balanced one, it can be transformed into a synchronous d-q-o frame by applying Park's transformation. The matrix form (3) is given as follows:

$$
\frac{d}{d t}\left[\begin{array}{l}
I_{p d} \\
I_{p q}
\end{array}\right]=\left[\begin{array}{cc}
-r_{p} / L_{p} & +\omega \\
-\omega & -r_{p} / L_{p}
\end{array}\right]\left[\begin{array}{l}
I_{p d} \\
I_{p q}
\end{array}\right]+\left[\begin{array}{cc}
1 / L_{p} & 0 \\
0 & 1 / L_{p}
\end{array}\right]\left[\begin{array}{c}
V_{p d}-V_{c d}-V_{r d} \\
V_{p q}-V_{c q}-V_{r q}
\end{array}\right]
$$

\subsection{The modeling of the UPFC continues branch}

For the DC-side circuit, based on the power balance equation in the output and input of UPFC, the net real power exchanged by both the converters through DC side should be zero to keep the capacitor voltage constant [13]. The DC voltage $V_{d c}$ dynamics across the capacitor is given by the following equation

$$
\frac{d V_{d c}}{d t}=\frac{1}{C V_{d c}}\left(P_{e}-P_{e p}\right)
$$

With:

$P_{e}=v_{c a} i_{s a}+v_{c b} i_{s b}+v_{c c} i_{s c}$

$P_{p e}=v_{p a} i_{p a}+v_{p b} i_{p b}+v_{p c} i_{p c}$

With:

$P_{e}$ : active power absorbed of the $\mathrm{AC}$ system $P_{e p}$ : active power injected with the shunt inverter AC system.

By performing Park transformation, the DC voltage $V_{d c}$ dynamics across the capacitor can be described by the following equations.

$$
\frac{d v_{d c}}{d t}=\frac{2}{2 C V_{d c}}\left(v_{p d} I_{p d}+v_{p q} I_{p q}-v_{c d} I_{r d}-v_{c q} I_{r q}\right)
$$

\section{CONTROL STRATEGY OF THE UPFC}

\subsection{Control of the parallel converter}

The ordinary working principle of the parallel compensation of STATCOM is described as follows: active power control which means stabling the capacitor voltage of the DC side; reactive power control which means stabling the terminal voltage [14]. According to the system of (2) the control strategy of parallel compensation (STATCOM) is decoupling of the two current loops control, to decrease the coupling between the active and reactive powers. Block diagram of the control system is shown by Figure 3 . 


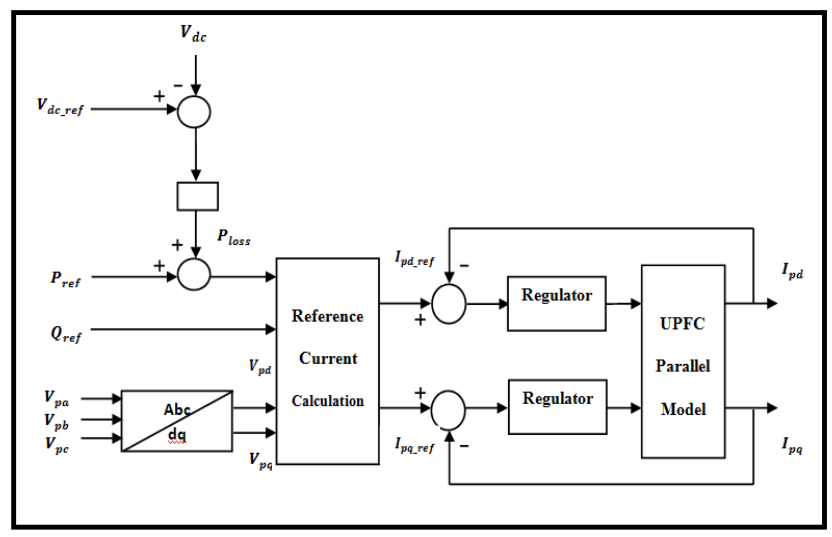

Figure 3. Control system of shunt part

\subsection{Control of the series converter}

The SSSC regulate the active and reactive power flow on the transmission line where the UPFC is installed by injection voltage of which the amplitude and the phase both can be adjusted. The control strategy of the series compensator is decoupling of the two current loops control. The diagram of control circuits of SSSC is given in the Figure 4. In this part, we have decided to evaluate the performances of the UPFC by two diverse controllers: PI and SMC.

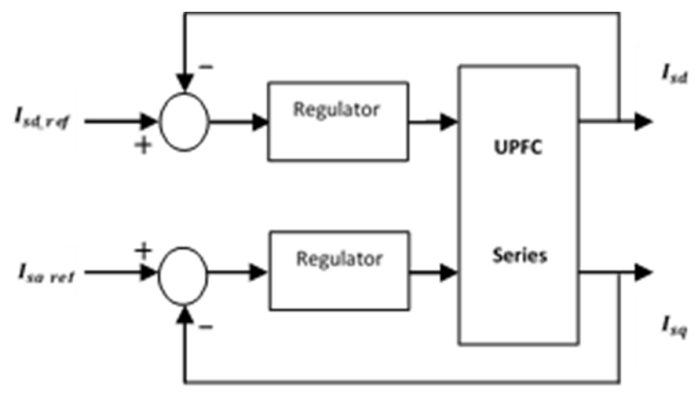

Figure 4. Control system of series part

\subsection{PI controller}

The PI controller is easy to develop. Figure 5 illustrates the two loops of our system (current and voltage), controlled by this type of regulator. In this figure, $k_{p}$ and $k_{i}$ successively represent proportional and integral gains of the PI controllers. These two gains are calculated using the pole compensation method where the system response time has been chosen equal to $\tau=5 \mathrm{~ms}$. The calculated terms are given in Table 1.

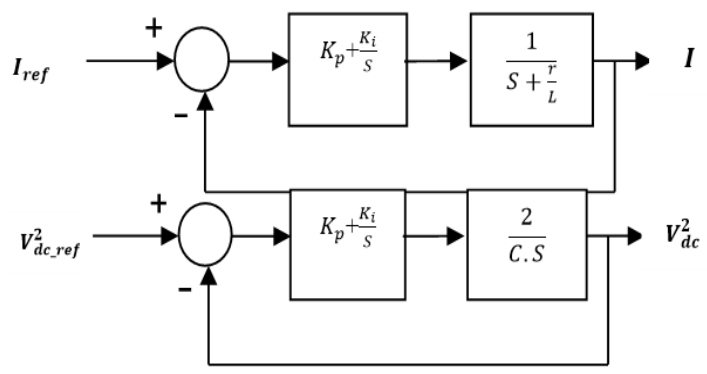

Figure 5. System with PI controller 
Table 1. Optimal parameters of the proposed controllers

\begin{tabular}{cccc}
\hline & Controller series part & Controller shunt part & Controller continues branch \\
\hline$K_{p}$ & $\frac{1}{\tau}$ & $\frac{1}{\tau}$ & $\frac{C}{2} \cdot \omega^{2}$ \\
$K_{i}$ & $\left(\frac{R}{L}\right)^{2} K_{p}$ & $\left(\frac{r_{p}}{L_{p}}\right) K_{p}$ & $C \cdot \xi \omega$ \\
\hline
\end{tabular}

It should be noted that the pole compensation method is not the only method existing in the literature for calculating the gains of the PI regulator. But it was used because of its simplicity.

\subsection{Sliding mode controller (SMC)}

$\mathrm{SMC}$ is one of the effective non linear robust control approaches since it provides system dynamics by an invariance property to uncertainties once the system dynamics are controlled in the sliding mode [15-17]. The main feature of SMC is that it just requires driving the error to a switching surface it consists of three parts Figure 6.

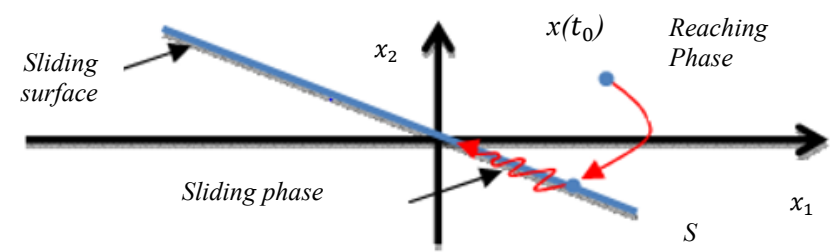

Figure 6. Phase portrait of SMC

\section{a. The switching surface choice}

In order to allow the application of the command by sliding mode, it is mandatory that the controlled system must be written in the following canonical form [18].

$$
\left\{\begin{array}{c}
\&=f(x, t)+B(x, t) V(x, t) \\
x \in R n, V \in R m, \operatorname{ran}(B(x, t))=m
\end{array}\right.
$$

Where: $f(x, t) ; B(x, t)$ are two continuous and uncertain non-linear functions, supposed limited.

We take the general equation to determine the sliding surface, proposed by J.J. Slotine [19-20], given by

$$
S(X)=\left(\frac{d}{d t}+\lambda\right)^{n-1} e ; e=x^{*}-x
$$

Where: $e$ is the error on the signal to be adjusted; $\lambda$ : a positive coefficient; $n$ : system order; $x^{*}$ : desired signal; $x$ : state variable of the control signal.

\section{b. Convergence condition}

The convergence condition is defined by the Lyapunov equation [13]; it makes the surface attractive and invariant

$$
\boldsymbol{S} \cdot \dot{\boldsymbol{S}}<\mathbf{0}
$$

\section{c. Control Calculation}

The control algorithm is defined by the relation [15]

$$
V^{c o m}=V^{e q}+V^{n}
$$

Here $V^{n}$ is the correction factor, $V^{e q}$ is the equivalent control vector, $V^{\text {com }}$ is the control vector which must be calculated in order that the stability conditions for the selected control are satisfied.

\footnotetext{
Advanced control scheme of a unifiedpower flow controller using sliding mode control (Abdellatif Hinda)
} 


$$
\begin{aligned}
& V^{n}=K \operatorname{sat}(S(X) / \delta) \\
& \operatorname{sat}(S(X) / \delta)=\left\{\begin{array}{c}
\operatorname{sign}(S) \text { if }|S|>\delta \\
S / \delta \text { if }|S|<\delta
\end{array}\right.
\end{aligned}
$$

Here, sat $((S(x) / \delta)$ is the proposed saturation function, $\delta$ is the boundary layer thickness.

In our study, the errors between the references and measured $I_{d}$ and $I_{q}$ currents have been chosen as sliding mode surfaces, so we can write the following relation:

$$
\left\{\begin{array}{l}
s_{d}=I_{s d_{\_} r e f}-I_{s d} \\
s_{q}=I_{s q \_r e f}-I_{s q}
\end{array}\right.
$$

The first order derivate of (8), one obtains

$$
\left\{\begin{array}{l}
\dot{s}_{d}=\dot{I}_{s d \_r e f}-\dot{I}_{s d} \\
\dot{s}_{q}=\dot{I}_{s q \_r e f}-\dot{I}_{s q}
\end{array}\right.
$$

Taking its derivative and replacing it in the current $\dot{I}_{s d}$ and $\dot{I}_{s q}$ expression (2) we get

$$
\begin{gathered}
\dot{s}_{d}=\dot{I}_{s d_{-} r e f}-\omega I_{s q}+\frac{r}{L} I_{s d}-\frac{1}{l}\left(v_{s d}-v_{c d}-v_{r d}\right) \\
\dot{s}_{q}=\dot{I}_{s q_{-} r e f}+\omega I_{s d}+\frac{r}{L} I_{s q}-\frac{1}{L}\left(v_{s q}-v_{c q}-v_{r q}\right)
\end{gathered}
$$

Replacing the expression of $v_{c d}$ and $v_{c q}$ in (10) by their expressions given in (15), one obtains

$$
\left\{\begin{array}{l}
\dot{s}_{d}=\dot{I}_{s d_{r e f}}-\omega I_{s q}+\frac{r}{L} I_{s d}-\frac{1}{L}\left(v_{s d}-\left(v_{c d}^{n}+v_{c d}^{e q}\right)-v_{r d}\right) \\
\dot{s}_{q}=\dot{I}_{s q_{r e f}}+\omega I_{s d}+\frac{r}{L} I_{s q}-\frac{1}{L}\left(v_{s q}-\left(v_{c q}^{n}+v_{c q}^{e q}\right)-v_{r q}\right)
\end{array}\right.
$$

$\mathrm{I}_{\mathrm{qr}}$ will be the component of the control vector used to constraint the system to converge to $\mathrm{S}=0$. The control vector $\boldsymbol{v}^{\boldsymbol{e q}}$ is obtain with imposing $\dot{\boldsymbol{S}}=\mathbf{0}$ so the equivalent control components are given with the following expression

$$
\left\{\begin{array}{l}
v_{c d}^{e q}=-L \dot{I}_{s d_{r e f}}+L \omega I_{s q}-r I_{s d}+v_{s d}-v_{r d} \\
v_{c q}^{e q}=-L \dot{I}_{s q_{r e f}}-L \omega I_{s d}-r I_{s q}+v_{s q}-v_{r q}
\end{array}\right.
$$

Using the same procedures as for part shunt we get the following expression:

$$
\left\{\begin{array}{l}
v_{c d}^{e q}=-L_{p} \dot{I}_{p d_{-} r e f}+L_{p} \omega I_{p q}-r_{p} I_{p d}+v_{p d}-v_{r d} \\
v_{c q}^{e q}=-L_{p} \dot{I}_{p q_{-} r e f}-L_{p} \omega I_{p d}-r_{p} I_{p q}+v_{p q}-v_{r q}
\end{array}\right.
$$

To obtain high-quality performances, dynamic an commutation around the surface, the control vector is imposed as follows [17]

$$
v=v^{e q}+K \cdot \operatorname{sign}(S)
$$

The sliding mode will exist only if the following condition is met:

$$
S \cdot \&<0
$$

\section{SIMULATION RESULTS}

This session is devoted to the numerical simulation using MATLAB/SIMULINK of a UPFC coupled to a $220 \mathrm{~V} / 50 \mathrm{~Hz}$ grid. The parameters of the system used in the simulation are given in Table 2. Three simulation tests were performed in this session to examine the performance of the controllers used. 


\subsection{Reference tracking test}

The objective of this test has for goal the study of the two controllers (PI and SMC) behavior in reference tracking. Figure 7 shows the obtained results. As shown by this figure, we can notice that the powers follow almost perfectly their references for the two types of controllers with a very reduced response time for the SMC controller compared to the PI regulator.

\subsection{Robustness}

We tested the robustness of the used controllers for a variation of the reactance $X L$. The results presented in Figure 8 show that this variation presents a clear effect on the active and reactive powers for the system with PI controller contrary to the system with SMC where this variation have almost no effect on active and reactive powers. Thus, it can be concluded that this last is robust against this parameter variation.

\subsection{Sensitivity to a sub-voltage perturbation}

The purpose of this test is the evaluation of the controllers used againts a sub-voltage effect $(+50 \%)$, applied between the instants $t=0.6 \mathrm{~s}$ and $t=0.62 \mathrm{~s}$. The obtained simulation results are exposed in Figure 9. Through this figure, we notice that the applied disturbance (sub-voltage) caused an almost negligible effect on the power curves for the two types of controllers used. Ce résultat est intéressant pour le système UPFC qui peut être soumis à des effets pareils.

Table 2. UPFC parameters used for simulation

\begin{tabular}{lccc}
\hline \multicolumn{1}{c}{ Parameter name } & Symbol & Value & Unit \\
\hline Network voltage & $V_{r}$ & 220 & $V$ \\
Voltage of the receiver & $V_{s}$ & 220 & $V$ \\
DC voltage & $V_{d c}$ & 280 & $V$ \\
Network frequency & $f$ & 50 & $H Z$ \\
The capacity of the & $C$ & 2 & $m F$ \\
common circuit DC & & & \\
Inductance 1 & $L$ & 1.125 & $m H$ \\
Resistance 1 & $r$ & 100 & $\Omega$ \\
Inductance 2 & $L_{p}$ & 1.125 & $m H$ \\
Resistance 2 & $r_{p}$ & 100 & $\Omega$ \\
\hline
\end{tabular}
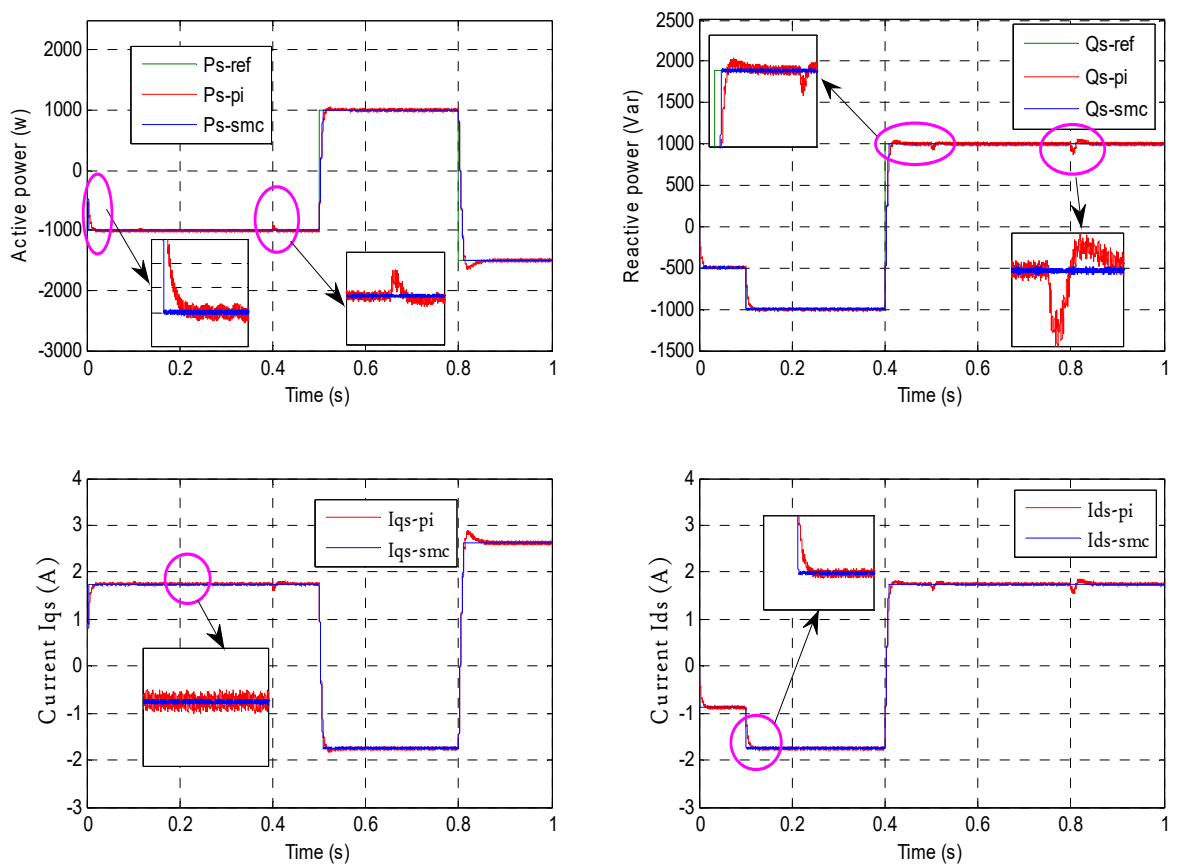

Figure 7. Reference tracking test 

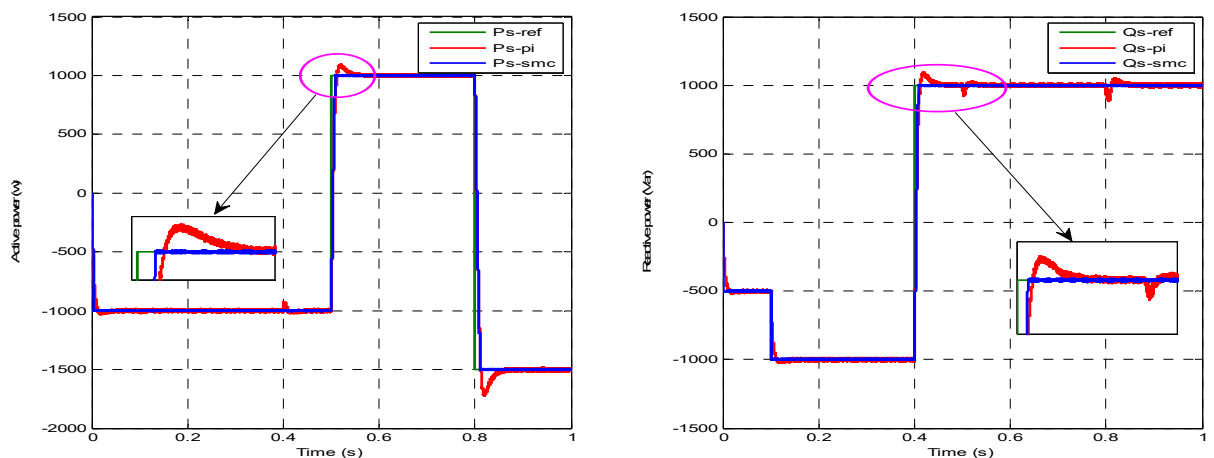

Figure 8 . Sensitivity to the reactance variation $(X L-30 \%)$
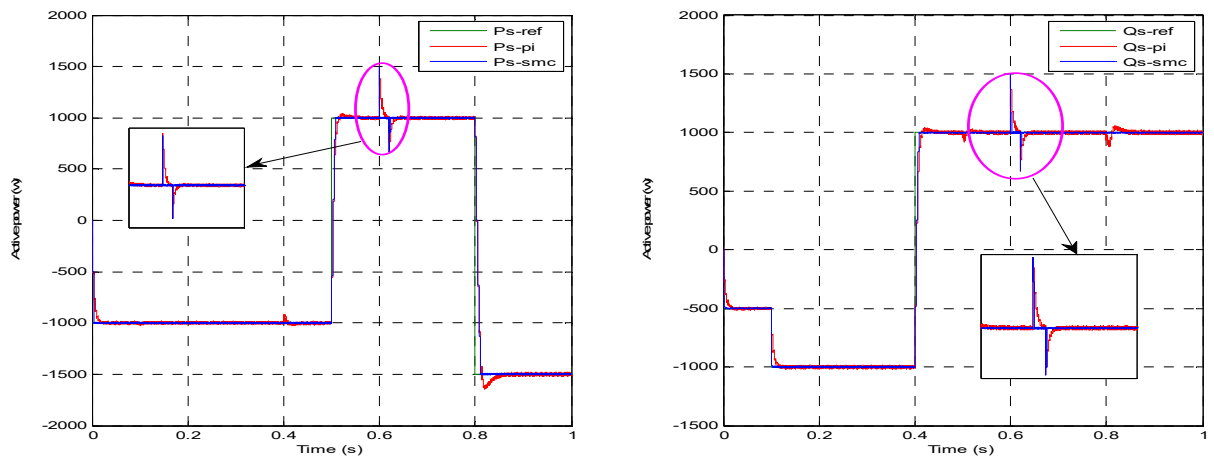

Figure 9. Sensitivity to sub-voltage perturbation $(+50 \%)$

\section{CONCLUSION}

This article was dedicated to the application of a robute control method based on the sliding mode for the control of a UPFC system. A comparative study by numerical simulation has clearly shown the superiority of the control used (SMC) compared to the conventional control based on PI regulators especially in the robustness test and the decoupling between active and reactive powers. Basing on the obtained results, the UPFC device based on SMC, can adjust the distribution of the system power flow between the transmission lines quickly and smoothly, and does not have a significant impact on the other operating parameters of the system.

\section{REFERENCES}

[1] G. Abdullah Salman, M. Hasan Ali, and A. Najim Abdullah, "Implementation Optimal Location and Sizing of UPFC on Iraqi Power System Grid (132 kV) Using Genetic Algorithm," International Journal of Power Electronics and Drive System (IJPEDS), vol. 9, no. 4, pp. 1607-1615, 2018.

[2] M. Boudiaf and M. Moudjahed, "Improvement of transient stability of power system by IPFC, SSSC and STATCOM," Journal of Electrical Engineering, vol. 14, no. 1, pp. 257-272, 2014.

[3] Yang, S., Liu Y., and Wang, X., Gunasekaran, D., Karki, U., and Fang Z. Peng, "Modulation and Control of Transformer-less UPFC," IEEE Transactions on Power Electronics, vol. 31, no. 2, pp. 1050-1063, 2016.

[4] Krishna, T. Murali, and Anjaneyulu, K. S. R., "Coordination of Intelligent Controllers for shunt and series converters of UPFC," Proceedings of the in ternational Conference on Power, Control, Communication and Computational Technologies for Sustainable Growth (PCCCTSG), pp. 11-14, 2015.

[5] Round S.D, yu Q, and Norum L.E, and T.M, "Performance of a Unified Power Flow Controller Using a D-Q Control System, AC and DC Power Transmission," Proceedings of the Sixth International Conference on AC and DC Power Transmission, vol. 423, pp. 357-362, 1996.

[6] Sharma N.K and Jagtap P.P, "Modelling and application of Unified Power Flow Controller (UPFC)," Third International Conference on Emerging Trends in Engineering and Technology (ICETET), pp. 350-355, 2010.

[7] Umre P.B, Bandal V.S, and Dhamne A.R, "Design of controller for Unified Power Flow Controller (UPFC) using Sliding Mode Control (SMC) strategies," In: the 5th In $\neg$ ternational Conference on Confluence The Next Generation Information Technology Summit (Confluence), pp. 893-897, 2014.

Int J Pow Elec \& Dri Syst, Vol. 11, No. 2, June 2020 : $625-633$ 
[8] Gorinevsky, D, Boyd, S, and Stein, G., "Robust terminal sliding mode power flow controller using unified power flow controller with adaptive observer and local measurement," IEEE IET Generation, Transmission \& Distribution, vol. 53, no. 10, pp. 1712-1723, 2014.

[9] Nayeripour, M, Narimani, M.S, and Niknam, T, and Jam, S., "Design of sliding mode controller for UPFC to improve power oscillation damping,"ELSEVIER Applied Soft Computing, vol. 11, no. 8, pp. 4766-4772, 2011.

[10] Ali Mousmi, Ahmed Abbou, Yassine El Houm, and Anass Bakouri, "Real time implementation of a super twisting control of a BLDC motor," International Journal of Electrical and Computer Engineering (IJECE), vol. 9, no. 4, pp. 3032-3040, 2019.

[11] Bouanane, A, Yahiaoui, and Merzoug, "Robust stability power in the transmission line with the use of a UPFC system and neural controllers based adaptive control," International Journal of Power Electronics and Drive System (IJPEDS), vol. 10, no. 3, pp.1281-1296, 2019.

[12] Kamel, S and Jurado, F., "Comparison of various UPFC models for power flow control," ELSEVIER Electric Power Systems Research, vol. 121, pp. 257-272, 2015.

[13] Mallick R.K., Nahak N., and Sinha R.R., "Fuzzy Sliding Mode Control for UPFC to Improve Transient Stability of Power System," Annual IEEE, India Conference (INDICON), New Delhi, India, pp. 1-6, 2015.

[14] Meng W and Qinxiang G., "Mathematical Model and Control Strategy of UPFC," In: Sixth International Conference on Measuring Technology and Mechatronics Automation (ICMTMA), Zhangjiajie, China, 2014.

[15] Wai R. J. and Chang J. M., "Implementation of robust wavelet-neural-network sliding-mode control for induction servo motor drive," IEEE Transactions on Industrial Electronics, vol. 50, no. 6, pp. 1317-1334, 2008.

[16] Utkin, V. I., "Sliding mode control design principles and applications to electric drives," IEEE Transactions on Industrial Electronics, vol. 40, no. 1, pp. 23-36, 1993.

[17] Astrom K. J. and Wittenmark B., Adaptive Control. New York: Addison-Wesley, 1995.

[18] Sun, T, Chen, Z, and Blaabjerg, F., "Flicker study on variable speed wind turbines with doubly fed induction generators," IEEE Transactions on Energy Conversion, vol. 20, no. 4, pp. 896-905, 2005.

[19] Slotine, J. J. E. and Li, W., Applied Nonlinear Control. Englewood Cliffs. NJ: Prentice-Hall, 1991.

[20] Slotine, J. J. E and Stein, "Sliding controller design for non-linear systems," IJC International Journal of Control, vol. 40, no. 2, pp. 421-434, 2007.

Advanced control scheme of a unifiedpower flow controller using sliding mode control (Abdellatif Hinda) 